\title{
Relativistic Cornell-type mechanism of exotic scalar resonances
}

\author{
A. M. Badalian, M. S. Lukashov, and Yu. A. Simonov ${ }^{(1)}$ \\ NRC “Kurchatov Institute"-ITEP, B. Cheremushkinskaya 25, Moscow 117218, Russia
}

(Received 6 August 2020; accepted 5 October 2020; published 13 November 2020)

\begin{abstract}
The physical masses of the lowest scalar mesons strongly disagree with the calculated $q \bar{q}$ pole values. It is the purpose of this paper to explain theoretically the unusual spectrum of scalar mesons for both the ground and excited states, using the known spectra of the corresponding $q \bar{q}$ states and their connection to the meson resonances. The well-known Cornell coupled-channel mechanism is exploited for this connection together with the quark-chiral Lagrangian without fitting parameters. In addition to the scalars previously obtained using this method, $f_{0}(500), f_{0}(980), a_{0}(980)$, we predict all ground and first excited scalar states, $f_{0}(500), f_{0}(980), a_{0}(980), a_{0}(1450), K_{0}^{*}(700), K_{0}^{*}(1430), f_{0}(1370), f_{0}(1710)$, which are in reasonably good agreement with experimental data.
\end{abstract}

DOI: 10.1103/PhysRevD.102.094012

\section{INTRODUCTION}

The QCD theory of hadrons has been a highly developed resource for treating hadron properties and has explained a majority of observed hadrons thus far [1]. Nevertheless, there exist hadronic objects, considered nonstandard or extra states, with the properties (e.g., the masses and widths) strongly differing from theoretical predictions (see [2] for exotic $X Y Z$ states in $c \bar{c}$ systems), and similar questions [1] occur in the realm of light scalar mesons, such as $f_{0}(500), f_{0}(980), a_{0}(980), K_{0}^{*}(700)$. They can hardly be associated with the lowest conventional $q \bar{q}$ scalars, for several reasons: (a) their masses are strongly displaced relative to expected $q \bar{q}$ masses, and (b) in some cases two observed scalar resonances can be identified with a single $q \bar{q}$ state with the same quantum numbers.

This situation (unusual compared to the vector meson case) was well described in 1995 [3]. Now, 25 years later, we have a much better understanding of this topic. Indeed, continuous efforts of the physical community have resulted in a large amount of information about the properties of the scalars and their decays and production (see [4-10] for reviews and analysis, and [11-13] for the most recent reviews). Theoretical approaches to the scalar spectrum include the tetraquark model [14], the chiral model [15], the molecular model [16], the QCD sum rules [17], and lattice calculations [18]. Our approach is based on several premises:

Published by the American Physical Society under the terms of the Creative Commons Attribution 4.0 International license. Further distribution of this work must maintain attribution to the author(s) and the published article's title, journal citation, and DOI. Funded by SCOAP .
(1) The primary poles are due to $q \bar{q}$ bound states, which are subject to interaction with meson-meson ( $m-m$ systems).

(2) This interaction can be deduced from the quarkchiral Lagrangian without free parameters.

(3) The coupled-channel interaction inside the $m-m$ systems can connect more than one resonance to one original $q \bar{q}$ pole.

Similar ideas are not new and have been investigated largely since 1995 in [19-25] with the proper formalism created in this field. As an example, in [19] Beveren et al. used the coupled-channel model for the meson-meson and quark-antiquark channels, interacting via a suggested potential form with oscillator-type confinement for $q \bar{q}$, and obtained a good agreement with experimental data for the scalar mesons, including $f_{0}(500)$. It required, however, the introduction of fitting parameters, defined by comparison with the data. A similar idea was used in [21], where the channel coupling was fitted separately for each scalar meson to obtain agreement with experimental data. As will be seen below, we construct the formalism for the $q \bar{q}$-meson-meson interaction, which allows us to calculate all parameters without a fitting procedure.

The additional poles due to $m-m$ interaction have been also introduced in the unitarized chiral perturbation theory (CPTh) [26-32], see also [11]. In principle these results can be obtained without using $\mathrm{CPTh}$, while, exploiting dispersive methods and data, one obtains a reasonable picture of $f_{0}(500)$ and other scalar resonances [33-35].

From these studies it has become evident that the phenomenon of low-lying scalar mesons like $f_{0}(500)$ is not a casual accident but rather a systematic result of the unitarized CPTh (see, e.g., [27] and the informative review [11]). However, one of the main problems was not fully resolved. Indeed, quark-antiquark theory with 
confinement, gluon exchange, and spin-dependent forces included [36-41] has given reasonable values for many mesons, e.g., for vector mesons, and the question arises as to why in the lowest scalar states the discrepancies amount to around 500-600 MeV. Moreover, if we believe that chiral theory alone can produce lowest scalars, then the following question arises: what can be done with the $q \bar{q}$ poles in the amplitude? Are they extra states or are they strongly displaced by some mechanism?

As one can see the main point is the relation between the accurate quark-gluon predictions for ideal scalar objects and realistic chiral-induced resonances observed in nature. One way is to start with scalar states in standard chiral theory and to connect it to the $q \bar{q}$ states in the large $N_{c}$ limit and beyond. A reasonable assumption was realized in the framework of the unitarized chiral model [11] and analyzed with the help of the $\frac{1}{N_{c}}$ expansion methods, where the intrinsic $q \bar{q}$ and chiral expanded meson-meson states display different behaviors. We shall discuss this topic below with an example of the present formalism and shall draw our conclusions in Sec. V.

Despite all of the effort and a large amount of information, the main problems underlined above have not yet been fully resolved, and in Table II of [1] the lowest scalar resonances are identified with $f_{0}(1370)$ for $I=0$ and $a_{0}(1450)$ for $I=1$, implying that the lowest ${ }^{3} P_{0} q \bar{q}$ pole is around (1.4-1.5) GeV, which contradicts numerous calculations in relativistic models [36-39].

We should stress that the relativistic FeynmanSchwinger formalism, taking into account both perturbative and nonperturbative $q \bar{q}$ interactions [37,39-41], as well as the methods of $[36,38]$, treating the full Regge trajectories, provide accurate predictions within $50-80 \mathrm{MeV}$ of the resonance masses in most nonchiral $I, J^{P}$ states and around 20-30 MeV for the $\rho$ meson [39] and heavy quarkonia [40] without open channels. At the same time, for the scalars these methods [36-39] provide discrepancies with data up to $500 \mathrm{MeV}$ and one may have two scalar resonances $f_{0}(500)$ and $f_{0}(980)$ for one $q \bar{q}$ pole at around $1 \mathrm{GeV}$.

In this paper our main purpose is to formulate an explicit formalism for theoretical calculations of the scalar ground and excited states, taking into account accurate values of the $q \bar{q}$ poles and explicit (without free parameters) $q \bar{q}$-meson-meson connection.

This paper is the development of a formalism constructed in [42] aimed at the treatment of all lowest scalar states, including all first excited scalars.

In [42] the basic formalism was combined to explain the possible connection of the basic $q \bar{q}$ poles to the scalar resonances $f_{0}(500), f_{0}(980), a_{0}(980)$ via the quark-chiral coefficients and the $q \bar{q}$-meson-meson channel-coupling interaction. In these calculations [42] one fitting parameter in all cases was used-the channel radius $\lambda$ in the $q \bar{q}$-meson-meson transition. In this paper we formulate the approach in more detail, finding the stationary point value for $\lambda$ (see Table IV). In this way we are calculating all masses and coefficients without fitting parameters, using for that purpose the explicit form of the $q \bar{q}$ wave functions to calculate all coefficients. As a result, as will be shown below, we succeed in calculating both the ground and first excited states of all scalar mesons, made of $u, d, s$ quarks.

In this paper, as well as in [42], for theoretical formulation of the scalar meson problem use is made of the relativistic method, which is similar to the nonrelativistic Cornell coupled-channel mechanism [43], developed for heavy quarkonia, where the charmonium states $c \bar{c}$ transform into the $D \bar{D}$ states and back many times, leading to a displacement of the resulting combined resonances. This displacement occurs via creation of a pair of light quarks and numerically is of the order or less than $50 \mathrm{MeV}$. Later Eichten et al. studied quantitatively displaced resonances in charmonium [44], and Simonov and colleagues used the Cornell formalism to study both charmonium and bottomonium systems [45].

The general theory of channel-coupled (CC) resonances was developed in [46] in a general form, not assuming pole structures in any channel, while the $\mathrm{CC}$ resonance can occur, as in the case of the $\Upsilon(n S) \pi$ system coupled to $B B^{*}$ or $B^{*} B^{*}$ (see the final reference in [45]). Below we shall be specifically interested in the $q \bar{q}$ poles found in the relativistic path-integral formalism coupled to a pair of chiral mesons.

One of the basic points of this method is derivation of the transition elements between the $q \bar{q}$ and the meson-meson systems, and below we use, as in [42], the chiral confining Lagrangian (CCL) [47-51]. In this Lagrangian both $q \bar{q}$ and the chiral degrees of freedom (d.o.f.) are connected with known coefficients and one can immediately find the coupling coefficient, which defines the decay or transformation probability of several mesons $(1,2,3, \ldots)$ into $q \bar{q}$ or vice versa. This is the fact which we shall use below and which shall enable us to find strong displacements of $\pi \pi$ and $\pi \eta$ resonances and much smaller values for $K \bar{K}$.

The formalism of CCL allows one to obtain standard relations like Gell-Mann-Oakes-Renner (GMOR) [52] and also to extend the possibilities of the standard chiral formalism; e.g., it allows one to calculate all coupling constants between $q \bar{q}$ and two or more chiral mesons and, in particular, to numerically calculate the decay constants $f_{\pi}, f_{K}$, etc. [53].

In this paper our purpose is to exploit the exact positions of the $q \bar{q}$ poles, defined in relativistic theory (see $[37,39]$ and the references therein), and establish explicit relations between the known ${ }^{3} P_{0} q \bar{q}$ state characteristics and resulting new resonance pole parameters, which we shall call the pole projection mechanism (PPM).

In the framework of PPM, as shown in [42], a single $q \bar{q}$ pole can create one projected resonance, one for a given $\phi \phi$ (meson-meson) channel, coupled to the $q \bar{q}$ channel. Including the $\phi \phi$ channel coupling (e.g., 
in $\pi \pi-K \bar{K}$ channels), one obtains two resonances connected with one $q \bar{q}$ pole. This mechanism was applied in the case of the $f_{0}(500)$ and $f_{0}(980)$ resonances [42], when from the original $q \bar{q}$ pole with the mass $M_{1}=$ $1.05 \mathrm{GeV}$ two resonances, $f_{0}(500)$ and $f_{0}(980)$, are created. In this way both properties mentioned above were demonstrated, since $f_{0}(500)$ occurs due to the $\pi \pi$ channel coupling to the $q \bar{q}$ initial state with the mass $M_{1}$, while $f_{0}(980)$ appears due to the $K \bar{K}-q \bar{q}$ channel coupling. Simultaneously, in the case with isospin $I=1$ and the initial mass $M_{1}$, the $q \bar{q}$ pole is coupled to both channels, $\pi \eta$ and $K \bar{K}$, and produces two resonances near $1 \mathrm{GeV}$, which can be associated with $a_{0}(980)$.

As shown in [42], in the PPM exists the only variable parameter-the spatial radius $\lambda$ of the quark-meson transition amplitude, denoted as $k^{(I)}(q \bar{q}, \varphi \varphi)$, which varies in the range $(0.2-0.3) \mathrm{fm}$. In this paper the parameter is fixed in the following way. The spatial radius $\lambda$ enters the quarkchiral Lagrangian [47-50] in the mass parameter $M(\lambda)=\sigma \lambda$, and in the case of $\pi, K$ mesons it is fixed by the calculation of the decay constants $f_{\pi}, f_{K}$ [53], which yields $\lambda=0.83 \mathrm{GeV}^{-1}$. In the $q \bar{q}-\varphi \varphi$ transition case we calculate for the first time dependence of the coefficient $k^{(I)}(q \bar{q}, \varphi \varphi)$ on $\lambda$ and find a stable maximum at $\lambda=\lambda_{0}$ in the region $\left(1 \leq \lambda_{0} \leq 1.5\right) \mathrm{GeV}^{-1}$ (see Table IV). This is taken as a basic point of our method, yielding the fixed value of $k^{(I)}\left(\lambda_{0}\right)$ and the fixed $\lambda=\lambda_{0}$. Since the string tension $\sigma$ is known to be equal to $0.18 \mathrm{GeV}^{2}$, the meson and quark masses are fixed, $\lambda$ at the stationary point is equal $1.5 \mathrm{GeV}^{-1}=0.30 \mathrm{fm}$, and in this way all parameters of our formalism are fixed and known.

In this paper we further extend PPM theory to include the radial excitations of the $q \bar{q}$ states and find the resulting scalar resonances. To this end we consider the $n \bar{n}, n \bar{s}, s \bar{s}$ states with $n_{r}=0,1$ and $I=0,1 / 2,1$ and show that the inclusion of the radial excited $q \bar{q}$ pole makes the PPM even more pronounced, when the lower pole, coupled with the meson-meson channels, has large shift down, while the second highest pole has a much smaller shift. In this way we demonstrate the important visible feature of the scalar resonances: the lowest $n_{r}=0$ poles are much more strongly shifted than the $n_{r}=1$ poles.

To calculate the resulting shifted poles we need (1) the transition coefficients $k^{(I)}\left(\lambda_{0}\right)$, discussed above; (2) the $q \bar{q}$ pole masses $M_{1}, M_{2}$, computed in the framework of relativistic path-integral Green's functions [54]; and (3) the free $\varphi \varphi$ Green's functions $G_{\varphi \varphi}\left(E, \lambda_{0}\right)$, defined as the spatial distance $\lambda_{0}$ between the in and out $\varphi \varphi$ states. As a result, we find the complex energy poles, corresponding to observed resonances $f_{0}(500), f_{0}(980), f_{0}(1370), f_{0}(1500), a_{0}(980)$, $a_{0}(1450), K_{0}^{*}(700), K_{0}^{*}(1430), f_{0}(1710)$.

The plan of the paper is as follows. In Sec. II we present the details of the PPM formalism of [42] in the case of the $I=0,1 / 2,1 ; J^{P}=0^{+}$channels, and in Sec. III we analyze the dynamics of our theory and calculate the resulting positions of the resonances. The inclusion of radial excited $q \bar{q}$ states and calculation of the resulting scalar resonances are done in Sec. IV. Section V is devoted to a discussion of the results and possible future developments of our approach.

\section{THE QUARK-CHIRAL DYNAMICS IN THE $(q \bar{q})$-(MESON-MESON) CHANNEL}

The main element of the Cornell formalism [43] is the expression for the total quark-meson Green's function (resolvent) $\mathcal{G}(E)$ via the $q \bar{q}$ resolvent $G_{q \bar{q}}$ and the meson-meson resolvent $G_{\varphi \varphi}$,

$$
\mathcal{G}(E)=\frac{A}{1-V_{q \varphi} G_{\varphi \varphi}(E) V_{\varphi q} G_{q \bar{q}}(E)},
$$

so the resonance energies are to be found by using the equation

$$
V G_{\varphi \varphi}(E) V G_{q \bar{q}}(E)=1,
$$

where the main point is the transition element $V_{q \varphi}=V_{\varphi q}^{+}$.

In $[43,44]$ it was shown how the channel coupling affects the charmonium poles. Later on this formalism has acquired the specific features necessary for explaining the poles in the heavy-quark systems, e.g., in $X(3872)$ [45], where the original $2^{3} P_{1}$ pole of the $c \bar{c}$ system is strongly shifted due to transitions of $c \bar{c}\left(2^{3} P_{1}\right)$ into the $D \bar{D}^{*}$ meson-meson state and back, which finally provides a pole at the $D \bar{D}^{*}$ threshold. Actually the equation for the position of the new quark-meson pole has similar forms: nonrelativistic [43-45] and relativistic in the new formulations for the scalars [42].

$G_{\varphi \varphi}(E) \Gamma G_{q \bar{q}}(E) \Gamma=1$, where $\Gamma$ is the $q \bar{q}-\varphi \varphi$ transition vertex, and in [42] it was found that for the chiral $\varphi \varphi$ mesons the value $\Gamma$ is large in the case of $\pi \pi$ and $\pi \eta$ systems.

Note that one could call $X(3872)$ as the $D \bar{D}^{*}$ resonance, but at the same time it can be considered as the shifted $c \bar{c}$ resonance, implying that it is the combined $c \bar{c}-D \bar{D}^{*}$ phenomenon, or the $c \bar{c}$ pole projected on the $D \bar{D}^{*}$ channel.

At this point one realizes that a single $c \bar{c}$ pole can interact with one of the $\left(D \bar{D}, D \bar{D}^{*}, D^{*} \bar{D}^{*}\right)$ states and can be strongly connected with one resonance. In the heavyquarkonia case the resulting pole shifts are of the order of $\sim 50 \mathrm{MeV}$ if the meson-meson thresholds are near the original $Q \bar{Q}$ poles, whereas in the general case the situation they can be different and, as shown in [42], in light mesons the pole shifts can reach $500 \mathrm{MeV}$. At this point it is important to stress the general features of the PPM method when the original $(q \bar{q})$ pole is projected onto the mesonmeson pole due to the interaction between the $q \bar{q}$ and chiral meson-meson channels, implying a strong but 
meson-dependent coupling. As a result, one $q \bar{q}$ pole can be projected originally into one meson-meson resonance, associated with the corresponding meson-meson threshold, and later, taking into account the meson-meson channel coupling, can be connected with two or more resonances. As shown in [42], this happens in the case of the $f_{0}(500)$ (the $\pi \pi$ channel) and the $f_{0}(980)$ (the $K \bar{K}$ plus coupled $\pi \pi)$, which are both connected to the $n \bar{n}\left(1^{3} P_{0}\right)$ pole at around $1.1 \mathrm{GeV}$.

These features create a completely new picture of possible "extra poles," generated by the regular $q \bar{q}$ poles in QCD, not connected to any molecular or tetraquark mechanisms. Note that the PPM can easily be extended to the three-meson decays $\left(m_{1}, m_{2}, m_{3}\right)$, coupled to the $q \bar{q}$ pole, as it occurs in the cases with the isospin $I=1$, $J=1,2$, namely, the $a_{1}(1 P), a_{2}(1 P)$ cases, which will be discussed elsewhere.

Below we shall present the PPM, which can explain the appearance of a new pole for each new meson-meson combination, starting with one original $q \bar{q}$ pole, as was done in the $f_{0}(500), f_{0}(980)$ case. We start with the basic element of the PPM formalism in the case of chiral mesons - the CCL, introduced in [47-49] and extended recently in [50]. This Lagrangian is a generalization of the standard chiral theory, which takes into account not only chiral meson but also the quark-antiquark d.o.f. The latter are necessary to calculate the meson coupling constants $\left(f_{\pi}, f_{K}, \ldots\right)$ [53], to write the correct Green's functions for chiral mesons, and also to calculate the higher $O\left(p^{4}, p^{6}\right)$ terms of CPTh (see [50]).

The CCL has the form

$$
L_{\mathrm{CCL}}=-N_{c} \operatorname{tr} \log \left(\hat{\partial}+\hat{m}+s_{0}+\hat{s}+M \hat{U}\right),
$$

where $\hat{U}$ is the standard chiral operator,

$$
\begin{gathered}
\hat{U}=\exp \left(i \gamma_{5} \hat{\varphi}\right), \quad \hat{\varphi}=\frac{\varphi_{a} \lambda_{a}}{f_{a}}, \\
\hat{\varphi}=\sqrt{2}\left(\begin{array}{ccc}
\frac{1}{f_{\pi}}\left(\frac{\eta}{\sqrt{6}}+\frac{\pi^{0}}{\sqrt{2}}\right), & \frac{\pi^{+}}{f_{\pi}}, & \frac{K^{+}}{f_{K}} \\
\frac{\pi^{-}}{f_{\pi}} & \left(\frac{\eta}{\sqrt{6}}-\frac{\pi^{0}}{\sqrt{2}}\right) \frac{1}{f_{\pi}}, & \frac{K^{0}}{f_{K^{0}}} \\
\frac{K^{-}}{f_{K}}, & \frac{\bar{K}^{0}}{f_{K^{0}}}, & -\frac{2 \eta}{\sqrt{6} f_{\pi}}
\end{array}\right) .
\end{gathered}
$$

Note that the CCL plays the role of the generating functional, which can produce several interesting expansions. Indeed, exploiting the trace logarithmic structure of it, which allows one to separate a common factor, the CCL can be transformed into the following expression [50],

$$
L_{\mathrm{CCL}}=-N_{c} \operatorname{tr} \log (1-\eta) \text {, }
$$

where

$$
\eta=\hat{U}^{+} S^{-1}(\hat{\partial}+\hat{m})(\hat{U}-1),
$$

and for $m=0$ it gives an expansion in quark loops with the quark propagators $S$, which yields $O\left(p^{n}\right)$ terms, while the expansion in $\hat{m}$ to the second order yields the GMOR relations. In what follows we shall not use this type of expansion but instead exploit Eq. (5) as it is, expanding $\hat{U}$ in powers of $\varphi$, keeping the second order for the mesonmeson amplitude.

In Eq. (5) $M$ is the $q \bar{q}$ interaction term, $M=\sigma r$, which gives confinement interaction between $q$ and $\bar{q}$ everywhere in the $q \bar{q}$ loop; however, in the vertex, where chiral mesons of $\hat{U}$ are emitted, $M$ is multiplied by the operators $\varphi$. In this case, i.e., in the one- $\pi$, or the one- $K$, emission vertex, the value of $M$, as shown in [53], is equal to $M(\lambda)=0.15 \mathrm{GeV}$, which corresponds to $\lambda \cong 0.166 \mathrm{fm}=0.83 \mathrm{GeV}^{-1}$. In our case, when two mesons are emitted, below we shall find $\lambda$ as the stationary point of the transition coefficient, which is equal $0.2 \mathrm{fm}=1 \mathrm{GeV}^{-1}$. It is interesting that this value coincides with the fundamental length of the QCD vacuum, known from using the field correlator method [55].

In the case of the one-meson emission vertex the value of $M=0.15 \mathrm{GeV}$ is exactly that, which correctly gives the pion and kaon decay constants, calculated in the framework of the CCL. From [53] one has

$$
\sqrt{2} f_{\pi}=138 \mathrm{MeV}, \quad \sqrt{2} f_{K}=165 \mathrm{MeV},
$$

which are in good agreement with experimental values [1], $\sqrt{f_{\pi}}=(130.7 \pm 0.1 \pm 0.36) \mathrm{MeV}, \sqrt{f_{K}}=(159.8 \pm 1.4 \pm$ $0.44) \mathrm{MeV}$.

The main idea of the quark-chiral approach [47-50] is that the scalar confining operator $M(\lambda)$, violating chiral symmetry, is augmented by the chiral operator $U(\hat{\varphi})$, which can emit any number of chiral mesons at the vertex of the $q \bar{q}$ operator.

Correspondingly, one can introduce the chiral-free $q \bar{q}$ Green's function from Eq. (3) with $U=1$, which we call $G_{q \bar{q}}$ (see Fig. 1), the free meson-meson Green's function $G_{\varphi \varphi}$ (see Fig. 2), and the transition element from $q \bar{q}$ to the

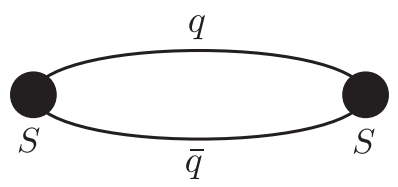

FIG. 1. The scalar $q \bar{q}$ Green's function $G_{q \bar{q}}$.

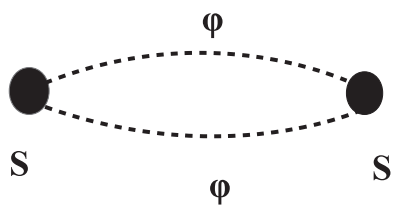

FIG. 2. The scalar $\varphi \varphi$ Green's function $G_{\varphi \varphi}$. 


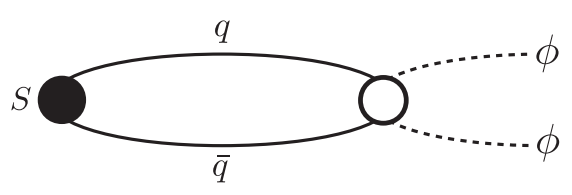

FIG. 3. The scalar $q \bar{q}$ Green's function with the emission of the chiral mesons.

$\varphi \varphi$ system, which is obtained from the CCL, Eq. (3), as shown in [42] (see Fig. 3):

$$
\Delta L=-N_{c} \operatorname{tr} \Lambda s \Lambda M(\lambda) \frac{\hat{\varphi}^{2}}{2} .
$$

Here $s$ is the external current, e.g., in the $f_{0}(500)$, $f_{0}(980)$ cases $(I=0)$ it is equal to 1 , while $\Lambda$ is the quark propagator, $\Lambda=\left(\hat{\partial}+m_{q}+M\right)^{-1}$.

At this point we can find the form of the $q \bar{q}$ Green's function augmented by the transition to the $\phi \phi$ system, which is needed to start the chain of transformations discussed here. This structure is presented in the next figure shown in Fig. 4.

As seen in Eq. (8) and following [42], one can find the numerical coefficient $C_{\varphi \varphi}^{(I)}$ in the transition factor $k^{(I)}(q \bar{q} \mid \varphi \varphi)$, which defines how many $\varphi \varphi$ are produced by the one $q \bar{q}$ state. In [42] this was done for isospin $I=0,1$. Here we shall also consider the case of the $K \pi$ channel $(I=1 / 2)$.

We conclude this section with the explicit form of the $I=1 / 2$ isotopic current, producing $K \pi$ in the case of the $K_{0}^{*}(700)$ resonance:

$$
\begin{aligned}
& \operatorname{tr}\left(j(u \bar{s}) \frac{\hat{\varphi}^{2}}{2}\right)=K^{+} \frac{\pi^{0}}{\sqrt{2}}+K^{0} \pi^{+}, \\
& \operatorname{tr}\left(j(d \bar{s}) \frac{\hat{\varphi}^{2}}{2}\right)=K^{+} \pi^{0}-\frac{\pi^{0}}{\sqrt{2}} K^{0} .
\end{aligned}
$$

\section{DYNAMICS OF THE $q \bar{q}$ AND THE MESON-MESON SYSTEMS}

The structure of the transition operator $M \frac{\hat{\varphi}^{2}}{2}$ [Eq. (8)] requires a detailed investigation. In [42] it was understood that the free meson-meson Green's function, created and annihilated at local points, diverges logarithmically and should be replaced by the physically motivated mesonmeson Green's function, where the initial and final distances between mesons are defined dynamically, i.e., given by the effective distance $\lambda$, determined by using the stationary point of the transition coefficient (see Table IV). In this paper we shall follow the same line of reasoning and define the meson-meson Green's function with fixed spatial distance $\lambda$ between the mesons at the initial and final points.

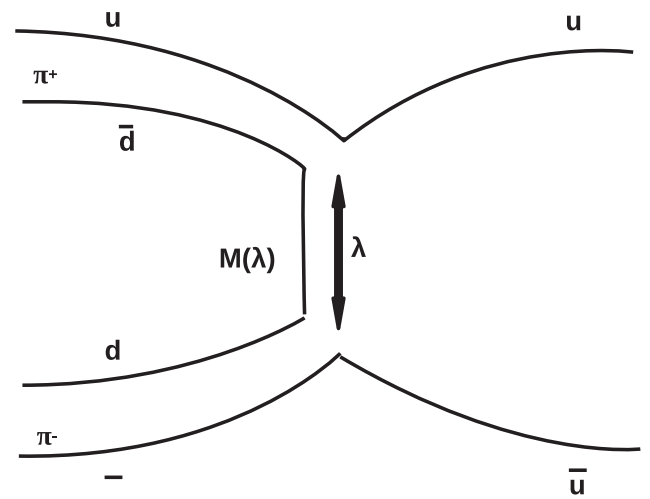

FIG. 4. The transition region $(q \bar{q} \mid \varphi \varphi)$ with the spatial distance $\lambda$ between the constituents.

One can start with the local $\varphi \varphi$ Green's function $G_{\varphi \varphi}(x, y)$, created using $\hat{\varphi}^{2}(x)$ in Eq. (8), and $G_{\varphi \varphi}(P)$ with the total momentum $P=(E, 0)$,

$$
G_{\varphi \varphi}(P)=\frac{1}{(2 \pi)^{4}} \int \frac{d^{4} p}{\left(p^{2}-m_{1}^{2}\right)\left((P-p)^{2}-m_{2}^{2}\right)} .
$$

To take into account nonlocality in the initial or final vertex we shall examine the structure of this nonlocal vertex in more detail, assuming its structure as shown in Fig. 4. As seen, for the distance $\lambda$ between $q$ and $\bar{q}$ (and effectively between $\varphi$ and $\varphi$ ) one should have the corresponding Green's functions $G_{q \bar{q}}$ and $G_{\varphi \varphi}$ of the form $G_{q \bar{q}}^{(\lambda)}\left(x, x^{\prime} \mid y, y^{\prime}\right), G_{\varphi \varphi}^{(\lambda)}\left(y, y^{\prime} \mid u, u^{\prime}\right)$ with the distance $\lambda=\left|\mathbf{x}-\mathbf{x}^{\prime}\right| \cong\left|\mathbf{y}-\mathbf{y}^{\prime}\right|$. The effective value of $\lambda$ in this vertex $(q \bar{q} \mid \varphi \varphi)$ is defined by the product $G_{q \bar{q}}^{(\lambda)} \sigma \lambda G_{\varphi \varphi}^{(\lambda)}$, which amounts to the $\lambda$ dependence of the transition coefficient and will be found in the following sections.

The Green's function $G_{\varphi \varphi}^{(\lambda)}\left(y, y^{\prime} \mid u, u^{\prime}\right)$ can be written as a product $\int \frac{d^{4} p}{(2 \pi)^{4}} f(p) \exp (i p(y-u)) \int \frac{d^{4} p^{\prime}}{(2 \pi)^{4}} f\left(p^{\prime}\right)$ $\exp \left(i p^{\prime}\left(y^{\prime}-u^{\prime}\right)\right)$. Now writing $\exp \left(i\left(p y+p^{\prime} y^{\prime}\right)\right)=$ $\exp \left(i\left(p+p^{\prime}\right)\left(y+y^{\prime}\right) / 2+i\left(p-p^{\prime}\right)\left(y-y^{\prime}\right) / 2\right) \quad$ with $P=\left(p+p^{\prime}\right)$, one can make the Fourier transformation in $\left(y+y^{\prime}\right) / 2$ while integrating over angles of the spatial vector $\left(y-y^{\prime}\right)$. The same arrangement can be done for $u, u^{\prime}$ vectors. Denoting $p-p^{\prime}=q$, one ends up with the same integral [Eq. (11)], multiplied by the square of the angular integral of $\exp (i \mathbf{q} p \lambda)$.

As it is, we need the explicit form of the meson-meson $G_{\varphi \varphi}^{(\lambda)}\left(y y^{\prime} \mid u u^{\prime}\right)$ and the $q \bar{q}$ Green's function $G_{q \bar{q}}^{(\lambda)}\left(x x^{\prime} \mid y y^{\prime}\right)$, defined with the initial and final spatial distances $\lambda$ between $\varphi$ and $\varphi$ or $q$ and $\bar{q}$. Since $G_{q \bar{q}}^{(\lambda)}$ is convergent at $\lambda=0$, we shall consider this effect later in this section and now start with the effect of spatial distance $\lambda$ in $G_{\varphi \varphi}^{(\lambda)}$. As shown above, the latter amounts to the angular integration of the factors $\exp \left(i p\left(u-u^{\prime}\right)\right)$ and $\exp \left(i p\left(y-y^{\prime}\right)\right)$, where we denote $q=p$. 
The result can be written in the form of the additional factor $F(\lambda p)=\left(\frac{\sin (\lambda p)}{\lambda p}\right)^{2}, p=|\boldsymbol{p}|$, appearing in Eq. (11), namely,

$$
G_{\varphi \varphi}^{(\lambda)}(P)=\frac{1}{(2 \pi)^{4}} \int \frac{d^{4} p F(\lambda p)}{\left(p^{2}-m_{1}^{2}\right)\left((P-p)^{2}-m_{2}^{2}\right)},
$$

where $F(\lambda p)=\left(\frac{\sin (\lambda p)}{\lambda p}\right)^{2}, p=|\boldsymbol{p}|$, appears to be due to averaging over directions of $\Delta \mathbf{y}=\mathbf{y}-\mathbf{y}^{\prime}, \Delta \mathbf{u}=\mathbf{u}-\mathbf{u}^{\prime}$, with $|\Delta \mathrm{y}|=|\Delta \mathrm{u}|=\lambda$.

The explicit form of Eq. (12) can be written in the c.m. frame,

$\operatorname{Re} G_{\varphi \varphi}^{(\lambda)}(E)=\int_{0}^{\infty} \frac{p^{2} d p}{4 \pi^{2}} \frac{F(\lambda p)}{\sqrt{p^{2}+m_{1}^{2}} \sqrt{p^{2}+m_{2}^{2}}}\left\{\frac{E\left(\sqrt{p^{2}+m_{1}^{2}}+\sqrt{p^{2}+m_{2}^{2}}\right)+m_{1}^{2}-m_{2}^{2}}{\left[\left(\sqrt{p^{2}+m_{1}^{2}}+\sqrt{p^{2}+m_{2}^{2}}\right)^{2}-E^{2}\right]\left[E+\sqrt{p^{2}+m_{1}^{2}}-\sqrt{p^{2}+m_{2}^{2}}\right]}\right\}$

$$
\operatorname{Im}_{\varphi \varphi}^{(\lambda)}(E)=\frac{F\left(\lambda p_{0}\right)}{16 \pi} \frac{\sqrt{\left[E^{2}-\left(m_{1}+m_{2}\right)^{2}\right]\left[E^{2}-\left(m_{1}-m_{2}\right)^{2}\right]}}{E^{2}},
$$

where $p_{0}$ is found from the relation $\sqrt{p_{0}^{2}+m_{1}^{2}}+$ $\sqrt{p_{0}^{2}+m_{2}^{2}}=E \geq m_{1}+m$.

Another way of the renormalization of $\operatorname{Re} G_{\varphi \varphi}(E)$ was accepted in [42], with $F(\lambda p) \rightarrow 1$ and the fixed upper limit of the $p$ integration, $p \leq N=1 / \lambda$. In what follows we shall compare both ways and find that they produce similar results. It is clear that the factor $F(\lambda p)$ is not introduced by hand but results from the $S$-wave angular integration of the product of the two-meson Green's functions at the spatial distance $\lambda$ from each other, which does not give rise to additional singularities. Note that $F(\lambda p)$ is actually a function of $\lambda^{2} p^{2}$ and therefore does not contribute to the difference $G_{\varphi \varphi}^{(\lambda)}(E+i \delta)-G_{\varphi \varphi}^{(\lambda)}(E-i \delta)$ on the cut $E \geq m_{1}+m_{2}$, and hence does not violate the unitarity condition.

In the case of the $K \pi$ Green's function one has $m_{1}=m_{K}$ (493 MeV for $K^{ \pm}$) and $m_{2}=m_{\pi} \cong 140 \mathrm{MeV}$. The resulting form [Eq. (13)] of the $\operatorname{Re} G_{\pi K}^{(\lambda)}(E)$ was computed numerically in the range $640 \mathrm{MeV} \leq E \leq 1200 \mathrm{MeV}$ for $\lambda=(0.5 ; 1 ; 2 ; 3) \mathrm{GeV}^{-1}$. The results of calculations show that $\operatorname{Re} G_{\pi K}^{(\lambda)}(E)$ is almost constant in the range $[0.64 \div 0,9]$. For the following we shall need the values of $\operatorname{Re} G_{\pi K}^{(\lambda)}$ at the point $E=0.64$ and $0.8 \mathrm{GeV}$ given in Table I.

In the right column of Table I the values of $\operatorname{Re} G_{\pi K}^{(\lambda)}(E=$ $640 \mathrm{MeV}$ ) are obtained with the cutoff of the integral over $d p$ in Eq. (13) at $N=1 / \lambda$. One can see their close values, within (10-15)\% accuracy, in columns 1 and 3.

Now we turn to the $q \bar{q}$ Green's function and shall use the same formalism for the $(n \bar{s})$ system as in [42] for the $(n \bar{n})$ system; for that, one can exploit the calculated positions of the $(n \bar{n})$ pole (see Table II) and, analogously, the $(s \bar{s})$ and $n \bar{s}$ poles. To calculate the $q \bar{q}$ Green's function and the $q \bar{q}$ eigenvalues we use, as in [42], the exact relativistic formalism (see [54] for a review and references based on the field correlator method [55]). This yields the relativistic Hamiltonian in the c.m. frame, containing the quark and antiquark kinetic energies $\omega_{1}, \omega_{2}$,

$H\left(\omega_{1}, \omega_{2}, \boldsymbol{p}\right)=\sum_{i=1,2} \frac{\boldsymbol{p}+\omega_{i}^{2}+m_{i}^{2}}{2 \omega_{i}}+V_{0}(r)+V_{s 0}(r)+V_{t}$.

Now one has two options for defining $\omega_{i}$ : (1) minimize $H\left(\omega_{1}, \omega_{2}, \boldsymbol{p}\right)$ in the values of $\omega_{1}, \omega_{2}$, which leads to the so-called spinless Salpeter equation (SSE), which is widely used (see, e.g., [36]), or (2) calculate the eigenvalue of Eq. (14) $E\left(\omega_{1}, \omega_{2}\right)$ and then to find its minimum (the socalled einbein approximation (EA); see [37,39,54] for details). The comparison of these approximations for the cases of $n \bar{n}$ scalar meson masses is given in Table II.

The interaction terms $V_{0}, V_{\text {so }}, V_{t}$ are the instantaneous potential with the scalar confinement $V_{0}$, perturbative and nonperturbative spin-orbit interactions $V_{\mathrm{so}}$, and tensor interaction $V_{t}$, which define the center-of-gravity eigenvalue $M_{\operatorname{cog}}(n P)$, the spin-orbit correction $a_{\text {so }}(n P)$, and the

TABLE I. The real part of the $K \pi$ Green's function as a function of the spacial distance $\lambda$ for two values of the energy, $E=640$ and $800 \mathrm{MeV}$.

\begin{tabular}{lccc}
\hline \hline $\begin{array}{l}\lambda \\
\left(\mathrm{GeV}^{-1}\right)\end{array}$ & $\begin{array}{c}\operatorname{Re} G_{\pi K}^{(\lambda)} \\
(640 \mathrm{MeV})\end{array}$ & $\begin{array}{c}\operatorname{Re} G_{\pi K}^{(\lambda)} \\
(800 \mathrm{MeV})\end{array}$ & $\begin{array}{c}\operatorname{Re} G_{\pi K}^{(\lambda)} \\
(640 \mathrm{MeV}, \text { cutoff })\end{array}$ \\
\hline 0.5 & 0.033 & 0.028 & 0.03 \\
1 & 0.025 & 0.02 & 0.022 \\
1.5 & 0.02 & 0.0165 & 0.017 \\
2 & 0.017 & 0.013 & 0.013 \\
3 & 0.013 & 0.007 & \\
\hline \hline
\end{tabular}


TABLE II. The masses (in MeV) of the $1^{3} P_{0}$ and $2^{3} P_{0} n \bar{n}$ states, obtained in the SSE, EA, and RT (the Regge trajectory formalism) by Badalian and Bakker [37,39], Ebert et al. [38], and Godfrey and Isgur [36].

\begin{tabular}{lccccc}
\hline \hline & \multicolumn{3}{c}{ BB [37,39] } & & \\
\cline { 2 - 4 } State & SSE & EA & RT & EFG [38] & GI [36] \\
\hline$n \bar{n} 1^{3} P_{0}$ & 1050 & 1093 & 1038 & 1176 & 1090 \\
$2^{3} P_{0}$ & 1461 & 1594 & 1435 & 1679 & 1780 \\
\hline \hline
\end{tabular}

tensor correction $c_{t}(n P)$. For the masses of the $n^{3} P_{0}$ states one has [37,39]

$$
M\left(n^{3} P_{0}\right)=M_{\operatorname{cog}}\left(n^{3} P_{0}\right)-2 a_{\mathrm{so}}-c_{t}
$$

The resulting masses of the $n \bar{n}, n \bar{s}, s \bar{s}$ states are given in Table II.

As shown in $[54,55]$, the $q \bar{q}$ Green's function can be written as a sum over the pole terms. As in [42], the lowest pole contribution to the $(q \bar{q})$ Green's function $G_{q \bar{q}}(E)$ can be written as

$$
G_{q \bar{q}}(E)=\sum_{n=1}^{\infty} \frac{\left(f_{s}^{(n)}\right)^{2} M_{n}^{2}}{M_{n}^{2}-E^{2}}=\frac{\left(f_{s}^{(1)}\right)^{2} M_{1}^{2}}{M_{1}^{2}-E^{2}}+\ldots
$$

where $f_{s}^{(1)}$ is as calculated in the $(n \bar{n})$ case in [42], while for all $q \bar{q}$ states it is as given in the Appendix A, and within $10 \%$ accuracy it has the value $f_{s}^{(1)} \cong 100 \mathrm{MeV}$, whereas the mass $M_{1}(n \bar{s})$ is obtained to be $M_{1}=(1210 \div 1240) \mathrm{MeV}$, and $M_{1}(s \bar{s}) \cong 1400 \mathrm{MeV}$; see Table III.

Now we can write the final equation for the position of the pole, resulting from the infinite series of the $(q \bar{q}) \rightarrow(\varphi \varphi) \rightarrow(q \bar{q}) \rightarrow \ldots$ transformations, in the same way as in [42].

$$
E^{2}=M_{1}^{2}\left\{1-k^{(I)}(q \bar{q} \mid \varphi \varphi)\left(\operatorname{Re} G_{\varphi \varphi}^{(\lambda)}(E)+i \operatorname{Im} G_{\varphi \varphi}^{(\lambda)}(E)\right)\right\}
$$

where

TABLE III. The masses (in $\mathrm{MeV}$ ) of the $n^{3} P_{0} q \bar{q}$ scalars, obtained using the method of [37,39], and their experimental values in the $\pi \pi, K \bar{K}, \pi \eta, \pi K$ systems.

\begin{tabular}{cccccc}
\hline \hline$n_{r}$ & & $n \bar{n}(I=1)$ & $n \bar{n}(I=0)$ & $n \bar{s}\left(I=\frac{1}{2}\right)$ & $s \bar{s}(I=0)$ \\
\hline 0 & $M_{1}$ & 1.050 & 1050 & 1240 & 1400 \\
& $\exp$ & $a_{0}(980)$ & $f_{0}(980), f_{0}(500)$ & $K_{0}^{*}(700)$ & $\tilde{f}_{0}(1370)$ \\
1 & $M_{2}$ & 1500 & 1500 & 1550 & 1740 \\
& $\exp$ & $a_{0}(1450)$ & $f_{0}(1500)$ & $K_{0}^{*}(1430)$ & $\tilde{f}_{0}(1710)$ \\
\hline \hline
\end{tabular}

$$
k^{(I)}\left(q \bar{q} \mid \varphi_{1} \varphi_{2}\right)=\frac{C_{i}^{2} M^{2}(\lambda)\left(f_{s}^{(1)}\right)^{2}}{f_{\varphi_{1}}^{2} f_{\varphi_{2}}^{2}}
$$

As seen below, coefficient $k^{(I)}$ plays the most important role in calculations of the resulting masses of scalar mesons. Moreover, its role is decisive in the definition of the nature of the resulting resonance, using the $\frac{1}{N_{c}}$ arguments (see Chaps. 4.1, 4.2, and 4.3 in [11,27]). As shown in [53], the meson decay constants $f_{s}, f_{\varphi}$ are proportional to $\sqrt{N_{c}}$, and therefore $k^{(I)}$ is $O\left(\frac{1}{N_{c}}\right)$. Then in our approach at large $N_{c}$ one is left with the bare $q \bar{q}$ poles and no other poles are present, and no connection to other objects. Moreover, the meson-meson interaction is not taken into account at this stage, while this is considered as the higher $\frac{1}{N_{c}}$ corrections, taken into account in the last stage of the method. The role of the $\pi \pi$ interaction can be seen, comparing the solid and broken lines in Fig. 5 of [42], where the latter takes this interaction into account in the framework of the dispersive method. One can notice that they are close at higher $E$ values but differ at smaller $E$ values, which implies that one needs the $\pi \pi$ interaction to comply with experiment, and, indeed, properly modifying the $\pi \pi$ Green's function one can reproduce the data based curve. This approach is different from the unitarized CPTh (see $[11,27]$, where the chiral-loop produced scalar mesons appear from the very beginning. We shall compare the two approaches in Sec. V.

At this point it is interesting to discuss the position of the poles, which are the self-consistent solutions of Eq. (18). First we consider the simplest case with equal masses of two mesons, $m_{1}=m_{2}$ and $E^{2}=p^{2}+4 m^{2}$, and start by solving Eq. (18) in terms of the variable $p$, taking into account the fact that $\operatorname{Re} G$ is a constant and $\operatorname{Im} G$ is proportional to $p, \operatorname{Im} G=p f\left(p^{2}\right)$. As a result, one obtains the equation for the position of the resonance in terms of $p$ :

$$
p^{2}+i p f\left(p^{2}\right)-p_{0}^{2}=0
$$

As a first approximation one can take $f\left(p^{2}\right)=f\left(p_{0}^{2}\right)=$ $f_{0}$ and, solving the quadratic equation, one obtains

$$
p=-i f_{0} / 2 \pm \sqrt{\left(f_{0}\right)^{2} / 4+p_{0}^{2}},
$$

which explicitly shows that the pole is on the second sheet with respect to the $2 m$ threshold. In next approximations one takes into account, step by step, the $p$ dependence of $f\left(p^{2}\right)$, observing the motion of the pole on the second sheet.

In Eq. (19) $C_{i}^{2}$ can be found for the $\pi \pi, K \bar{K}, \pi \eta$ cases as in [42], and from Eqs. (9) and (10), and for the $\pi K$ system it is equal to 
TABLE IV. The dependence of the ratio of the transition factor $k^{I}(q \bar{q} \mid \varphi \varphi) / k_{\max }^{(I)}$ on the spatial contact distance $\lambda$.

\begin{tabular}{lcccc}
\hline \hline$\lambda\left(\mathrm{GeV}^{-1}\right)$ & 0.5 & 1 & 1.5 & 2 \\
\hline$X(\lambda)$ & 0.29 & 0.816 & 1 & 0.04 \\
\hline \hline
\end{tabular}

TABLE V. The transition factor $k^{(I)}(q \bar{q} \mid \varphi \varphi)$ at $\lambda=1 \mathrm{GeV}^{-1}$ and $\lambda=1.5 \mathrm{GeV}^{-1}$ for different channels.

\begin{tabular}{lccccc}
\hline \hline$k(q \bar{q} \mid \varphi \varphi)$ & $(n \bar{n} \mid \pi \pi)$ & $(n \bar{n} \mid K K)$ & $(n \bar{n} \mid \pi \eta)$ & $(n \bar{s} \mid \pi K)$ & $(s \bar{s} \mid K K)$ \\
\hline$\lambda=1 \mathrm{GeV}^{-1}$ & 18.44 & 4.02 & 3.0 & 14.2 & 3.0 \\
$\lambda=1.5 \mathrm{GeV}^{-1}$ & 41.51 & 9.05 & 6.72 & 31.2 & 6.75 \\
\hline \hline
\end{tabular}

$$
C_{i}^{2}=\left(1+\frac{1}{\sqrt{2}}\right)^{2}=\frac{3}{2}+\sqrt{2}=2.91 \approx 3,
$$

while the pseudoscalar decay constants $f_{i}$ are known from [53] and experimental and lattice data,

$f_{K}=111 \mathrm{MeV}, \quad f_{\pi}=93 \mathrm{MeV}, \quad f_{\eta}=120 \mathrm{MeV}$.

The quark decay constants of the scalar mesons $f_{s}^{(i)}$ are calculated via the radial derivative of the $q \bar{q}$ wave function, as shown in Appendix A, with the values given in Table VIII. In Appendix B we show that $f_{s}^{i)}$ are strongly dependent on the value of $\lambda$ and that the effective region of $\lambda$ is inside the range $0 \leq \lambda \leq 1.5 \mathrm{GeV}^{-1}$. At the same time another factor in Eq. (19) $M^{2}(\lambda)$ grows with $\lambda$, so the optimal values of $\lambda$ can be obtained from the ratio $\frac{k^{(I)}(q \bar{q} \mid \varphi \varphi)}{k_{\max }^{(l)}(q \bar{q} \mid \varphi \varphi)} \equiv X(\lambda)$ given in Table IV.

Then, taking into account that $M(\lambda)=\sigma \lambda=$ $0.18 \mathrm{GeV}^{2} \cdot \lambda$, one has the following values of the transition factors $k^{(I)}(q \bar{q} \mid \varphi \varphi)$ at $\lambda=1 \mathrm{GeV}^{-1}$ and $\lambda=$ $1.5 \mathrm{GeV}^{-1}$ (see Table V).

Using these values of $k^{(I)}(\bar{q} \bar{q} \mid \varphi \varphi)$ in Eq. (18) and the values of $M_{1}$ from Table III, one obtains the parameters of the resonances in the channels $\pi \pi, K K, \pi \eta \pi K$ given in Table VI.

In Table VI one can see that the suggested PPM yields a reasonable picture of the resulting resonances in all $\varphi \varphi$ channels, and the differences between the calculated and observed resonance characteristics $(R, \Gamma)$ are of the order of indeterminacy intervals. A possible sign of disagreement seems to be in the $f_{0}(500)$ resonance, where PPM gives a resonance position some $150-200 \mathrm{MeV}$ above the experimental value. As discussed in [42], this fact implies that the $\pi \pi$ interaction in the $\pi \pi$ Green's function, $G_{\pi \pi}(E)$, has to be used to account for the low energy region, $E \lesssim 500 \mathrm{MeV}$. Indeed, accurate analysis based on the dispersive method in [56] confirms the $f_{0}(500)$ pole position at $E=(457-i 279) \mathrm{MeV}$, which is close to $E_{\exp }$, and for $f_{0}(980)$ it was obtained at $E=$ (996 $\pm 7-i 25 \mathrm{pm} 8) \mathrm{MeV}$, which is close to the experimental data. In the unitary chiral approach [26] for $a_{0}(980)$ the pole position was obtained at $E=$ $(937-i 85) \mathrm{MeV}$, while in [25] the two-pole method yields $E=970-i 45 \mathrm{MeV}$. In a similar approach in [24] it was not possible to find the position of $K *_{0}$ (700). We would like to stress here that our results for the poles of the scalar nonet in Table VI are obtained in a two-step process without a meson-meson interaction, which shifts the $f_{0}(500)$ pole down. As a result, one can see in Table VI for $\lambda=(1,1.5) \mathrm{GeV}^{-1}$

$$
E(\mathrm{GeV})=(0.85 \div 0.64)-i(0.17 \div 0.54)
$$

which differs from $E_{\text {exp }}$ by $200-400 \mathrm{MeV}$, while for the $f_{0}(980)$ our results are comparable to the data.

TABLE VI. The resonances in the channels $\pi \pi, K \bar{K}, \pi \eta, \pi K$, coupled at the distance $1 \mathrm{GeV}^{-1}$ and $\lambda=1.5 \mathrm{GeV}^{-1}$ to the $q \bar{q}$ poles $(n \bar{n}, n \bar{s}, s \bar{s})$, in comparison with experimental PDG data.

\begin{tabular}{ccccccc}
\hline \hline & $(q \bar{q} \mid \varphi \varphi)$ & $(n \bar{n} \mid \pi \pi)$ & $(n \bar{n} \mid K \bar{K})$ & $(n \bar{n} \mid \pi \eta)$ & $(n \bar{s} \mid \pi K)$ & $(s \overline{\mid} \mid K \bar{K})$ \\
\hline$\lambda=1$ & $k(q \bar{q} \mid \varphi \varphi)$ & 18.44 & 4.02 & 3.0 & 14.2 & 3.0 \\
& $\operatorname{Re} G_{\varphi \varphi}$ & 0.02 & 0.011 & 0.02 & 0.025 & 0.011 \\
& $\operatorname{Im} G_{\varphi \varphi}$ & 0.015 & 0.02 & 0.015 & 0.015 & 0.02 \\
& $\operatorname{Re} a, \operatorname{Im} a$ & $0.38 ; 0.276$ & $0.045+\mathrm{i} 0.08$ & $0.06+\mathrm{i} 0.045$ & $0.36+\mathrm{i} 0.213$ & $0.033+\mathrm{i} 0.06$ \\
& $E$ & $0.85-\mathrm{i} 0.17$ & $1.025-\mathrm{i} 0.044$ & $1.02-\mathrm{i} 0.025$ & $0.714-\mathrm{i} 0.078$ & $1.37-\mathrm{i} 0.041$ \\
& $k(q \bar{q} \mid \varphi \varphi)$ & 41.51 & 9.05 & 6.75 & 31.2 & 6.75 \\
& $\operatorname{Re} G_{\varphi \varphi}$ & 0.015 & 0.018 & 0.018 & 0.0165 & 0.018 \\
& $\operatorname{Im} G_{\varphi \varphi}$ & 0.0155 & 0.015 & 0.015 & 0.015 & 0.015 \\
& $\operatorname{Re} a, \operatorname{Im} a$ & $0.645 ; 0.645$ & $0.162+\mathrm{i} 0.136$ & $0.1215+\mathrm{i} 0.10$ & $0.52+\mathrm{i} 0.468$ & $0.12+\mathrm{i} 0.10$ \\
& $E$ & $0.64-\mathrm{i} 0.54$ & $0.966-\mathrm{i} 0.08$ & $0.98-\mathrm{i} 0.056$ & $0.75-\mathrm{i} 0.21$ & $1.31-\mathrm{i} 0.074$ \\
& $E_{\mathrm{PDG}}$ & $0.400-0.550$ & 0.990 & 0.980 & $0.630-0.730$ & $1.200-1.500$ \\
& $\Gamma_{\mathrm{PDG}}$ & $0.400-0.700$ & $0.010-0.100$ & $0.050-0.100$ & $0.478(50)$ & $0.200-0.500$ \\
\hline \hline
\end{tabular}




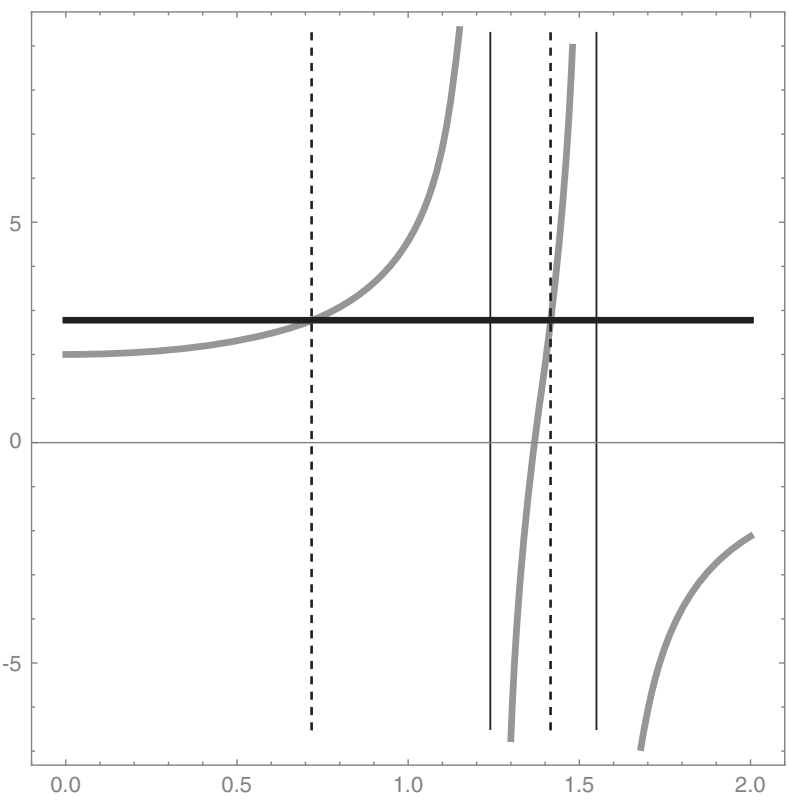

FIG. 5. The function $f(E)$ is shown with thick gray lines, with two poles at $E=M_{1}, M_{2}$ (shown with thin vertical lines). The intersection of $f(E)$ with the horizontal line at $a^{-1}=1 / 0.36$ yields the two resulting poles $E=E_{1}, E_{2}$, marked by vertical dashed lines.

\section{THE CASE OF TWO $q \bar{q}$ POLES}

Thus far we have studied the lowest ${ }^{3} P_{0}$ quark-antiquark poles, which due to the PPM are shifted down from the original position of around (1000-1400) $\mathrm{MeV}$ to the final position in the range (700-1300) $\mathrm{MeV}$, which can be associated with the lowest exotic resonances. However, in the $(n \bar{n})$ channel there is the radially excited pole $0^{++}$, $I=0$ at the initial position $M_{1}=(1490-1500) \mathrm{MeV}$, which can also be shifted down and have a position at around $1400 \mathrm{MeV}$, known as $f_{0}(1500)$. Also in the $K_{0}^{*}$ channel $\left(J^{P C}=0^{++}, I=\frac{1}{2}\right)$ there exists a higher resonance, coupled to the same $K \pi$ decay channel, $K_{0}^{*}(1430)$, which can originate from the radial excited $(n \bar{s})$ pole at $M_{2}=1550 \mathrm{MeV}$. Below we shall show a remarkable property of the PPM, where the shift down of the lowest $(q \bar{q})$ pole changes a little if the radial excitations are taken into account, while the mass shift of the higher $(q \bar{q})$ pole is strongly suppressed compared to the ground state. This property of the level repulsion follows from the structure of the PPM equations themselves.

Indeed, writing the one-channel, one-pole PPM [Eq. (35)] in the same form as in [42], one has

$$
G_{\varphi \varphi}(E) k^{(I)}(q \bar{q} \mid \varphi \varphi) \frac{M_{1}^{2}}{M_{1}^{2}-E^{2}}=1
$$

with

$k^{(I)}(q \bar{q} \mid \varphi \varphi)=\frac{\left(C_{\varphi \varphi}^{(I)}\right)^{2} M^{2}(\lambda)\left(f_{s}^{(1)}\right)^{2}}{f_{\varphi}^{4}}, \quad f_{\varphi}=f_{\pi}, f_{K}, f_{\eta}$.

This equation can be generalized while including the radially excited pole $M_{2}$ as follows:

$$
G_{\varphi \varphi}(E)\left[k_{1}^{(I)}(q \bar{q} \mid \varphi \varphi) \frac{M_{1}^{2}}{M_{1}^{2}-E^{2}}+k_{2}^{(I)}(q \bar{q} \mid \varphi \varphi) \frac{M_{2}^{2}}{M_{2}^{2}-E^{2}}\right]=1 .
$$

To better understand the situation with two projected poles we consider Eq. (27) and approximate $k_{1}^{(I)} \approx k_{2}^{(I)}$ (which holds in most cases according to Table VIII in Appendix A). From Eq. (27) one has the equation

TABLE VII. The scalar resonance positions and the widths in the two-pole formalism.

\begin{tabular}{lccccc}
\hline \hline$(q \bar{q} \mid \varphi \varphi)$ connection & $(n \bar{n} \mid \pi \pi)$ & $(n \bar{n} \mid K \bar{K})$ & $(n \bar{n} \mid \pi \eta)$ & $(n \bar{s} \mid \pi K)$ & $(s \bar{s} \mid K \bar{K})$ \\
\hline$n_{r}=0$ & 1.05 & 1.05 & 1.05 & 1.24 & 1.4 \\
$q \bar{q}$ mass $(\mathrm{GeV})$ & & & & & \\
$n_{r}=1$ & 1.50 & 1.5 & 1.5 & 1.55 & 1.74 \\
Transition coefficient $k^{(I)}(q \bar{q} \mid \varphi \varphi)$ & 18.44 & 4.02 & 3.0 & 14.2 & 3.0 \\
$a(E)=k^{(I)} G_{\varphi \varphi}(E)$ & $0.38+\mathrm{i} 0.28$ & $0.045+\mathrm{i} 0.08$ & $0.06+\mathrm{i} 0.045$ & $0.36+\mathrm{i} 0.213$ & $0.033+\mathrm{i} 0.06$ \\
$E_{1}\left(n_{r}=0\right)(\mathrm{GeV})$, & 0.8 & 1.04 & 1.02 & 0.85 & 1.36 \\
$\Gamma_{1}(\mathrm{MeV})$ & 980 & 32 & 40 & 640 & 72 \\
& $f_{0}(500)$ & $f_{0}(980)$ & $a_{0}(980)$ & $K_{0}^{*}(700)$ & $f_{0}(1370)$ \\
$E_{\mathrm{PDG}}^{(1)}(\mathrm{GeV})$ & $0.40-0.55$ & 0.99 & 0.98 & $0.63-0.73$ & $1.2 \div 1.5$ \\
$\Gamma_{\mathrm{PDG}}(\mathrm{MeV})$ & $400-700$ & $0.10-100$ & $0.50-100$ & 480 & $200 \div 500$ \\
$E_{2}\left(n_{r}=1\right)(\mathrm{GeV})$ & 1.28 & 1.45 & 1.45 & 1.4 & 1.72 \\
$\Gamma(\mathrm{MeV})$ & 100 & 84 & 52 & 40 & 76 \\
& $f_{0}(1370)$ & $f_{0}(1500)$ & $a_{0}(1450)$ & $K_{0}^{*}(1430)$ & $f_{0}(1710)$ \\
$E_{\mathrm{PDG}}^{(2)}$ & $1200-1500$ & 1.50 & 1.48 & 1.425 & 1.72 \\
$\Gamma(\mathrm{MeV})$ & $200 \div 500$ & $\Gamma=109$ & $\Gamma=265$ & $\Gamma=270$ & $\Gamma=120$ \\
\hline \hline
\end{tabular}


$f(E)=\frac{M_{1}^{2}}{M_{1}^{2}-E^{2}}+\frac{M_{2}^{2}}{M_{2}^{2}-E^{2}}=\frac{1}{k^{(I)} G_{\varphi \varphi}(E)}=a^{-1}$.

Then, taking case $(n \bar{s} \mid \pi K)$ as an example and neglecting $\operatorname{Im} G_{\varphi \varphi}$, from Table VI one obtains $a=k^{(1 / 2)} \operatorname{Re} G_{\pi K}=0.36$, and the resulting $f(E)$, as a function of $E$, has two poles defined by the intersection of the straight line $f(E)=\frac{1}{0.36}$ (see Fig. 5). In Fig. 5 one can easily see how the resulting poles $E_{1}, E_{2}$ are shifted relative to $M_{1}, M_{2}$, in the approximation of zero $\operatorname{Im} G_{\pi K}$.

To proceed with the case of $K_{0}^{*}(700), K_{0}^{*}(1430)$, we solve the quadratic in $E^{2}$ [Eq. (28)] with $M_{1}=1.24 \mathrm{GeV}$, $M_{2}=1.55 \mathrm{GeV}$ and obtain two approximate solutions for $\lambda=1 \mathrm{GeV}^{-1}$

$E_{1}=(0.78-i 0.33) \mathrm{GeV}, \quad E_{2}=(1.40-i 0.035) \mathrm{GeV}$.

These solutions correspond to the intersection points in Fig. 5 and were obtained by treating the imaginary part of $G_{\pi K}(E)$ as a perturbation. To take it fully into account one can write the solution of Eq. (28) as

$$
\begin{aligned}
E^{2}= & \frac{1}{2}\left(M_{1}^{2}+M_{2}^{2}\right)(1-a) \\
& \pm \sqrt{\frac{1}{4}\left(M_{1}^{2}+M_{2}^{2}\right)^{2}(1-a)^{2}-M_{1}^{2} M_{2}^{2}(1-2 a)}
\end{aligned}
$$

and use

$$
a=\operatorname{Re} a+i \operatorname{Im} a=k^{(I)}(q \bar{q} \mid \varphi \varphi)\left(\operatorname{Re} G_{\varphi \varphi}+i \operatorname{Im} G_{\varphi \varphi}\right)
$$

from Table VI in the case $(n \bar{s} \mid \pi K)$, calculated, e.g., for $\lambda=1 \mathrm{GeV}^{-1}$.

In a similar way one can consider all the cases: $(n \bar{n} \mid \pi \pi)$, $(n \bar{n} \mid K \bar{K}),(n \bar{n} \mid \pi \eta),(n \bar{s} \mid K \pi)$, and $(s \bar{s} \mid K \bar{K})$. The resulting pole positions for $\lambda=1 \mathrm{GeV}^{-1}$, generated by ground and radially excited scalar $q \bar{q}$ poles, are given in Table VII.

From Table VII one can see a reasonable agreement between the predicted and observed resonance characteristics, but with a few exclusions. The first one refers to the higher position of the predicted mass $f_{0}(500)$ with $E_{1}=800 \mathrm{MeV}$, with, however, a large width, which implies significant uncertainty in the resonance position and, as we discussed above, calls for the account of the $\pi \pi$ interaction in $G_{\pi \pi}$ at small energies. The second discrepancy might be more significant. Namely, the first $(s \bar{s} \mid K \bar{K})$ resonance occurs exactly at $1.37 \mathrm{GeV}$ (see Table VII) and could be associated with $f_{0}(1370)$; however, the latter prefers to decay into $\pi \pi, 4 \pi$ and the $K \bar{K}$ ratio is less than $10 \%$ [1].

At the same time the second $(n \bar{n} \mid \pi \pi)$ resonance is predicted to be at around $1.3 \mathrm{GeV}$ with the width
$\Gamma_{\pi \pi} \approx 100 \mathrm{MeV}$, and the $(n \bar{n} \mid K \bar{K})$ resonance to be at $1.45 \mathrm{GeV}$ with the width $\Gamma_{K \bar{K}} \approx 100 \mathrm{MeV}$; the latter has to be associated with $f_{0}(1500)$. Unfortunately $f_{0}(1500)$ decays mostly into $\pi \pi, 4 \pi$. Thus one faces three inconsistencies in the theory: $\pi \pi$ resonance at $1300 \mathrm{MeV}$ and two $K \bar{K}$ resonances at 1450 and $1360 \mathrm{MeV}$, while in experiment one has two resonances $f_{0}(1370)$ and $f_{0}(1500)$, decaying mostly into $\pi \pi$ and $4 \pi$.

Evidently, here appears a strong mixing pattern of three (or more) resonances, which can additionally be enlarged by the code mechanism $(K \bar{K} \mid n \bar{n}) \frac{M_{2}}{M_{2}^{*}-E^{2}}(n \bar{n} \mid \pi \pi)$ near the $n \bar{n}$ pole at $M_{2}=1.5 \mathrm{GeV}$. As an additional argument for this mixing and the resulting damping of the $K \bar{K}$ decay mode, one can use the small value of the $K \bar{K}$ decay width of $70 \mathrm{MeV}$ for the $(s \bar{s} \mid K \bar{K})$ resonance at $1.36 \mathrm{GeV}$, while the corresponding experimental resonance $f_{0}(1370)$ has a large $\pi \pi, 4 \pi$ width, $\Gamma=(200 \div 500) \mathrm{MeV}$. This interesting topic requires substantial analysis and a separate paper.

\section{CONCLUSIONS AND AN OUTLOOK}

In our paper we presented the simplest version of the CC mechanism with the code $(q \bar{q} \mid \varphi \varphi)$, which is the relativistic and chiral extension of the original Cornell code used for charmonium resonances [43]. This is the realization of the CC mechanism [46], where due to an infinite set of transformations of one system into another one can provide a pole (the bound state) in this set, even if both systems are free. Here the basic role is played by the magnitude of the transition amplitude. A concrete example of the resulting $Z_{b}$ resonances was given in the last references of [45].

It was also demonstrated that in the case of the scalar mesons the role of transition coefficient $k^{(I)}(q \bar{q} \mid \varphi \varphi)$ is extremely important since it can be a very large number, $k^{(0)}(n \bar{n} \mid \pi \pi) \sim 30 \pm 10$, in the $(n \bar{n} \mid \pi \pi)$ and $(n \bar{s} \mid K \pi)$ cases (see Table VI), and small number, $k \sim 1.0$, in the other cases. In Tables VI and VII one can see that this large range of changes helps us to understand the situation with the scalars, where the shifts of the resonances are so different in different $\varphi \varphi$ systems, and the maximal one is in the $(n \bar{n} \mid \pi \pi)$ case.

At this point one can see the main difference of the present approach from other existing formalisms. As explained in the paper, the connection between $q \bar{q}$ and the meson-meson channels plays the basic role, and starting from the single $q \bar{q}$ pole, one can define roughly the parameters of all lowest scalar resonances (this does not mean that other mechanisms are ruled out). However, here one must find the transition coefficients explicitly, without fitting parameters, which we could do with the use of the CCL and the stationary point in the function $k^{(I)}(\lambda)$. An approximate way of adjusting this connection was already used in the unitarized meson model [19-21], where one needs to introduce different parameters for each channel to describe the transitions. Another approach is the dispersive 
method [56], where the integral equations together with experimental data are used to fully define the meson-meson amplitudes and the positions of resonances. These results of [56] are very useful for performing the final adjustment of the positions of the lowest scalar resonances, obtained in our formalism without fitting parameters, as demonstrated in Fig. 5 of [42]. This is clearly seen by comparing our (solid line) curves of the real and imaginary parts of the $\pi \pi$ scattering amplitude with those (broken lines) curves obtained using the dispersive methods in [56]. Looking at the solid and broken lines in Fig. 5 of [42], one can notice that they are close together at higher $E$ but divergent at smaller $E$ values. This implies that one needs to include the $\pi \pi$ interaction to comply with experiment and, indeed, properly modifying the $\pi \pi$ Green's function, one can reproduce the data curve.

This can be compared to the unitarized chiral model approach $[27,28]$, where the $\frac{1}{N_{c}}$ analysis shows that the $q \bar{q}$ component is all important at large $N_{c}$ and can be considered the "seed" of the state. As $N_{c}$ is lowered, this state has an increased coupling to the $\pi \pi$ channel, and it is this channel that dominates its existence when $N_{c}=3^{\prime \prime}$ [28]. Therefore both approaches yield similar resulting pictures with intrinsically connected $q \bar{q}$ and experimentally found scalar resonances.

Another important feature of the PPM is the appearance of a new $\varphi \varphi$ resonance, created using a single $q \bar{q}$ pole- this $\varphi \varphi$ resonance can appear, in principle, in each $\varphi \varphi$ system connected with this $q \bar{q}$ pole. To have more resonances connected with the same $q \bar{q}$ pole, one needs an additional direct $\phi_{1} \phi_{1}-\phi_{2} \phi_{2}$ interaction. This happens for $\pi \pi$ and $K \bar{K}$ systems, where two resonances $f_{0}(500)$ and $f_{0}(980)$ are created in this way by the $q \bar{q}$ pole at $E=1050 \mathrm{MeV}$. Note that these resonances finally become connected due to the $\pi \pi-K \bar{K}$ channel coupling, and in some cases two nearby resonance poles can be located on different sheets, as observed in lattice analysis by Dudek et al. [18].

We have already stressed the important role of the $\varphi \varphi$ interaction in obtaining the correct position of lowest resonances $f_{0}(500)$ and $K_{0}^{*}(700)$. Actually our approach provides an alternative way to describe $\varphi \varphi$ scattering amplitudes when the $q \bar{q}$ dynamics is included at the first stage and the $q \bar{q}-\varphi \varphi$ transition is taken into account as a second step, and the final stage should include a detailed account of the $\varphi \varphi$ interaction. The comparison of the resulting $\pi \pi$ amplitude, using only the two first steps, with the realistic $\pi \pi$ data done in [42], shows exactly that the two-step amplitude roughly describes the main featuresthe extrema and zeros of the amplitude-but strongly distorts the amplitude at small energies, where the $\varphi \varphi$ interaction is important. To solve the scalar meson problem, as demonstrated above, the simplified two-step procedure was sufficient. On the other hand, the full three-step procedure provides the exact $\varphi \varphi$ amplitude with the correct $q \bar{q}$ input, as shown in [42].
At this point it is important to discuss in more detail the problem with the internal structure of resonances and its connections (and assignments) to different quark and meson channels. In the literature this topic is widely discussed in connection with $\frac{1}{N_{c}}$ expansion estimates; see, e.g., [57]. Here we start at another point and first discuss "elementary" one-channel bound states and resonances. It is clear that one-channel states represent the majority of the known states $(q \bar{q}, q q q$, atoms, atomic nuclei, etc.) and that they have necessarily admixed states in their Fock columns, appearing from the rescattering series, but these new states do not necessarily appear in the form of additional poles. Those general properties are not connected directly to the $\frac{1}{N_{c}}$ expansion.

Now we get closer to the nature of the lowest scalar mesons. In our method one starts with the basic $q \bar{q}$ states, which produce poles around $(1-1,1) \mathrm{GeV}$, and the next story is the motion of this pole down, due to interaction with the $\varphi \varphi$ components, or up in connection with, say, glueball states. To follow this line let us rewrite our basic equation (18) in a simpler form, assuming that the meson-meson Green's function $G_{\varphi \varphi}(E)=G(E)$ also has a pole term

$$
E^{2}=M^{2}\left(1-k^{(I)} G(E)\right), \quad G(E)=\frac{c}{E^{2}-m^{2}}+d .
$$

Solving for the quadratic equation in $E^{2}$, one obtains two roots which are attracting or repulsing each other depending on the signs of $c, d$. One can envisage different situations with the final positions of these poles, but it is clear that both poles survive somewhere on the next sheets of the $E$ plane. In our case the coefficient $k^{(I)}$ is large for $f_{0}$ and the original pole at $1 \mathrm{GeV}$ is strongly shifted down. The questions as to what happens with the pole of $G(E)$ and whether it exists at all are of secondary importance: if the data display only one pole below $1 \mathrm{GeV}$, one is inclined to associate it with the original $q \bar{q}$ pole. Note that in this approach the major role is played by the magnitude and the energy dependence of the channel coupling, which is assumed to be exactly given by theory. Summarizing, one can say that one can always associate the observable resonance with the initial elementary channel; however, the strong coupling to the meson-meson channel for $N_{c}=3$ makes this resonance a common property. Following this approach in our Table VII we have associated the resulting resonances with the $q \bar{q}$ components; however, their positions could be strongly displaced from the original masses, leaving the possibility of large non- $q \bar{q}$ admixtures in these states but keeping the role of the seed components in these states.

Another feature of the PPM found in this paper is the relatively small shifts of all radial excited resonances compared with the ground states, especially in the $(n \bar{n} \mid \pi \pi)$ and $(n \bar{s} \mid \pi K)$ cases. As a whole, we have explained the general features of the scalar meson spectrum, leaving 
the details of the $K \bar{K}-\pi \pi$ coupling to future works. The methods of this paper have a quite general applicability. In particular they can be used to calculate the positions of $a_{1}$, $a_{2}$ and other resonances containing $3 \pi, \rho-\pi$ constituents.

\section{ACKNOWLEDGMENTS}

The work of M. S. L. and Yu. A. S. is supported by the Russian Science Foundation in the framework of a scientific project, Grant No. 16-12-10414.

\section{APPENDIX A: DECAY CONSTANTS OF THE $n \bar{n}, n \bar{s}$, AND $s \bar{s}$ STATES}

As explained in [42], the $q \bar{q}$ Green's function is computed in the Fock-Schwinger formalism, based on the relativistic path-integral method. In this formalism the $q \bar{q}$ Green's function in the c.m. frame $(\mathbf{P}=0)$ has the form

$G_{q \bar{q}}(E)=\sum_{n} \frac{\left(f_{s}^{(n)}\right)^{2} M_{n}^{2}}{M_{n}^{2}-E^{2}} \rightarrow \frac{\left(f_{s}^{(1)}\right)^{2} M_{1}^{2}}{M_{1}^{2}-E^{2}}+\frac{\left(f_{s}^{(2)}\right)^{2} M_{2}^{2}}{M_{2}^{2}-E^{2}}$,

where $M_{n}, n=1,2$, are the energy eigenvalues, while $f_{s}^{(n)}$ are the $P$-wave decay constants, which are discussed and calculated in Appendix A of [42].

Here we only detail the explicit form of $f_{3}^{(1)}$ and its dependence on the quark masses and the radial quantum number $n$.
The explicit form of $f_{s}^{(n)}$ can be written as [42]

$$
\left(f_{s}^{(n)}\right)^{2}=\frac{2 N_{c}\left(R_{n P}^{\prime}(0)\right)^{2}}{4 \pi \omega_{n} \bar{\omega}_{n} M_{n}}
$$

where $\omega_{n}, \bar{\omega}_{n}$ are the average energies of the quark and the antiquark in the relativistic $q \bar{q}$ system obeyed by the confinement, the color Coulomb, and spin-dependent interactions [39]. Concrete calculations, done in this framework as in [42], bring the following results presented in Table VIII.

\section{APPENDIX B}

As shown in Eq. (A2), the decay constant $f_{s}^{(n)}$ (where $s$ is the scalar) is defined via the derivative $R_{n P}^{\prime}(0)$, while other factors in Eq. (A2) do not depend on $r$.

For the decay constant, defined at the spatial distance $r=\lambda$ between $q$ and $\bar{q}$ (see Fig. 4), the decay constant $f_{s}^{(n)}(\lambda)$ is determined via the derivative $R_{n P}^{\prime}(\lambda)$, i.e., generalizing Eq. (A2),

$$
\left(f_{s}^{(n)}(\lambda)\right)^{2}=\frac{2 N_{c}\left(R_{n P}^{\prime}(\lambda)\right)^{2}}{4 \pi \omega_{n} \bar{\omega}_{n} M_{n}} .
$$

The values of $R_{n P}^{\prime}(\lambda)$ have been computed numerically in the relativistic formalism of [37,39] and corresponding values of $R_{1 P}^{\prime}(\lambda),\left(R_{1 P}^{\prime}(\lambda)\right)^{2}$ are given in Table IX together with the ratios of the decay constants $\eta(\lambda)=\left|\frac{f_{s}(\lambda)}{f_{s}(0)}\right|^{2}$.

TABLE VIII. The quark kinetic energy $\omega_{i}$ (in $\mathrm{GeV}$ ), the derivative of the radial wave function at the origin $R_{i P}^{\prime}(0)\left(\mathrm{GeV}^{5 / 2}\right)$, the mass $M_{i}$ (in $\mathrm{GeV}$ ), and the decay constant $f_{s}^{(i)}$ for the ground state $(i=1)$ and the first excited state $(i=2)$.

\begin{tabular}{llllllcc}
\hline \hline$q \bar{q}$ & $\omega_{1} ; \omega_{2}$ & $R_{1 P}^{\prime}(0)$ & $R_{2 P}^{\prime}(0)$ & $M_{1}$ & $M_{2}$ & $\left(f_{s}^{(1)}\right)^{2}\left(\mathrm{GeV}^{2}\right)$ & $\left(f_{s}^{(2)}\right)^{2}\left(\mathrm{GeV}^{2}\right)$ \\
\hline$n \bar{n}$ & $0.48 ; 0.50$ & 0.0845 & 0.0906 & 1.05 & 1.5 & 0.0142 & 0.0103 \\
$n \bar{s}$ & $0.53 ; 0.56$ & 0.091 & 0.106 & 1.24 & $1.55 \div 1.61$ & 0.010 & 0.0108 \\
$s \bar{s}$ & $0.54 ; 0.57$ & 0.099 & 0.116 & 1.4 & 1.74 & 0.0112 & 0.0101 \\
\hline \hline
\end{tabular}

TABLE IX. The space distances $\lambda$, the derivative of the wave function $R_{1 P}^{\prime}(\lambda)$ and $\left(R_{1 P}^{\prime}(\lambda)\right)^{2}$, and the parameter $\eta(\lambda)$ for the ground $n \bar{n}$

\begin{tabular}{|c|c|c|c|c|c|c|c|c|}
\hline$\lambda\left(\mathrm{GeV}^{-1}\right)$ & 0.25 & 0.50 & 0.75 & 1.0 & 1.25 & 1.50 & 1.75 & 2.0 \\
\hline$R_{n P}^{\prime}(\lambda)\left(\mathrm{GeV}^{5 / 2}\right)$ & 0.0852 & 0.082 & 0.0764 & 0.0684 & 0.06 & 0.0504 & 0.0101 & 0.0077 \\
\hline$\left(R_{n P}^{\prime}(\lambda)\right)^{2} \mathrm{GeV}^{5}$ & 0.00726 & 0.00672 & 0.00583 & 0.00468 & 0.0036 & 0.0025 & 0.0001 & $5.9 \times 10^{-5}$ \\
\hline$\eta(\lambda)=\left|\frac{f_{s}^{(1)}(\lambda)}{f_{s}^{(1)}(0)}\right|^{2}$ & 0.98 & 0.91 & 0.79 & 0.63 & 0.486 & 0.343 & 0.0138 & 0.008 \\
\hline
\end{tabular}
state. 
[1] M. Tanabashi, K. Hagiwara, K. Hikasa et al. (Particle Data Group), Phys. Rev. D 98, 030001 (2018).

[2] N. Brambilla, S. Eidelman, C. Hanhart, A. Nefediev, C.-P. Shen, C. E. Thomas, A. Vairo, and C.-Z. Yuan, Phys. Rep. 873, 1 (2020).

[3] N. A. Törnqvist, Z. Phys. C 68, 647 (1995).

[4] F. E. Close and N. A. Törnqvist, J. Phys. G 28, R249 (2002).

[5] D. V. Bugg, Phys. Rep. 397, 257 (2004).

[6] C. Amsler and N. A. Törnqvist, Phys. Rep. 389, 61 (2004).

[7] R. L. Jaffe, Phys. Rep. 409, 1 (2005).

[8] M. R. Pennington, Int. J. Mod. Phys. A 21, 747 (2006).

[9] E. Klempt and A. Zaitsev, Phys. Rep. 454, 1 (2007).

[10] N. N. Achasov, Phys. Usp. 41, 1149 (1998); Nucl. Phys. A675, 279 (2000).

[11] J. R. Pelaez, Phys. Rep. 658, 1 (2016).

[12] G. Rupp and E. van Beveren, Acta Phys. Pol. B Proc. Suppl. 11, 455 (2018).

[13] N. N. Achasov and G. N. Shestakov, Phys. Usp. 62, 3 (2019).

[14] R. L. Jaffe, Phys. Rev. D 15, 267 (1977); 15, 281 (1977); G.'t Hooft, G. Isidori, L. Maiani, A. D. Polosa, and V. Riquer, Phys. Lett. B 662, 424 (2008); D. Ebert, R. N. Faustov, and V. O. Galkin, Eur. Phys. J. C 60, 273 (2009); G. Eichmann, C. Fischer, and W. Heupel, Phys. Lett. B 753, 282 (2016).

[15] G. Colangelo, J. Gasser, and H. Leutwyler, Nucl. Phys. B603, 125 (2001); I. Caprini, G. Colangelo, and H. Leutwyler, Phys. Rev. Lett. 96, 132001 (2006).

[16] J. D. Weinstein and N. Isgur, Phys. Rev. Lett. 48, 659 (1982); J. D. Weinstein and N. Isgur, Phys. Rev. D 27, 588 (1983); 41, 2236 (1990); J. A. Oller, E. Oset, and A. Ramos, Prog. Part. Nucl. Phys. 45, 157 (2000); E. Oset, W.-H. Liang, M. Bayar et al., Int. J. Mod. Phys. E 25, 1630001 (2016); Yu. S. Kalashnikova and A. V. Nefediev, Phys. Usp. 62, 568 (2019).

[17] Z. G. Wang, Eur. Phys. J. C 76, 427 (2016).

[18] M. G. Alford and R. L. Jaffe, Nucl. Phys. B578, 367 (2000); H. Suganuma, K. Tsumura, N. Ishii, and F. Okiharu, Prog. Theor. Phys. Suppl. 168, 168 (2007); N. Mathur, A. Alexandru, Y. Chen, S. J. Dong, T. Draper, I. Horváth, F. X. Lee, K. F. Liu, S. Tamhankar, and J. B. Zhang, Phys. Rev. D 76, 114505 (2007); M. Loan, Z. H. Luo, and Y. Y. Lam, Eur. Phys. J. C 57, 579 (2008); S. Prelovsek and D. Mohler, Phys. Rev. D 79, 014503 (2009); T. Kunihiro, S. Muroya, A. Nakamura, C. Nonaka, M. Sekiguchi, and H. Wada (SCALAR Collaboration), Phys. Rev. D 70, 034504 (2004); J. J. Dudek, R. G. Edwards, and D. J. Wilson (Hadron Spectrum Collaboration), Phys. Rev. D 93, 094506 (2016); D. Darvish, R. Brett, J. Bulava, J. Fallica, A. Hanlon, B. Hörz, and C. Morningstar, AIP Conf. Proc. 2249, 030021 (2020).

[19] E. van Beveren, T. A. Rijken, K. Metzger, C. Dullemond, G. Rupp, and J. E. Ribeiro, Z. Phys. C 30, 615 (1986).

[20] E. van Beveren and G. Rupp, Eur. Phys. J. C 22, 493 (2001).

[21] E. van Beveren, D. V. Bugg, F. Kleefeld, and G. Rupp, Phys. Lett. B 641, 265 (2006).

[22] N. A. Tornqvist and M. Roos, Phys. Rev. Lett. 76, 1575 (1996).

[23] N. A. Tornqvist, Z. Phys. C 68, 647 (1995); N. A. Tornqvist and M. Roos, Phys. Rev. Lett. 76, 1575 (1996).
[24] M. Boglione and M. R. Pennington, Phys. Rev. D 65, 114010 (2002).

[25] T. Wolkanowski, F. Giacosa, and D. H. Rischke, Phys. Rev. D 93, 014002 (2016).

[26] J. Oller and E. Oset, Phys. Rev. D 60, 074023 (1999).

[27] J. R. Pelaez and G. Rios, Phys. Rev. Lett. 97, 242002 (2006).

[28] J. Ruiz De Elvira, J. R. Pelaez, M. R. Pennington, and D. J. Wilson, Phys. Rev. D 84, 096006 (2011).

[29] J. Oller and E. Oset, Nucl. Phys. A620, 438 (1997).

[30] A. Dobado and J. Pelaez, Phys. Rev. D 56, 3057 (1997).

[31] A. Gomez Nicola and J. Pelaez, Phys. Rev. D 65, 054009 (2002).

[32] J. Pelaez, Phys. Rep. 658, 1 (2016).

[33] J. R. Pelaez and F. J. Yndurain, Phys. Rev. D 71, 074016 (2005); R. Garcia-Martin, R. Kaminski, J. R. Pelaez, and J. Ruiz de Elvira, Phys. Rev. Lett. 107, 072001 (2011).

[34] J. R. Pelaez, A. Rodas, and J. Ruiz De Elvira, Eur. Phys. J. C 79, 1008 (2019).

[35] R. Garcia-Martin, R. Kaminski, J. R. Pelaez, J. Ruiz De Elvira, and F. J. Yndurain, Phys. Rev. D 83, 074004 (2011).

[36] S. Godfrey and N. Isgur, Phys. Rev. D 32, 189 (1985).

[37] A. M. Badalian and B. L. G. Bakker, Phys. Rev. D 67, 071901 (2003).

[38] D. Ebert, R. N. Faustov, and V. O. Galkin, Phys. Rev. D 79, 114029 (2009).

[39] A. M. Badalian and B.L. G. Bakker, Phys. Rev. D 100, 034010 (2019).

[40] A. M. Badalian and B. L. G. Bakker, Phys. Rev. D 100, 054036 (2019).

[41] A. M. Badalian and B. L. G. Bakker, Few-Body Syst. 60, 58 (2019).

[42] M. S. Lukashov and Yu. A. Simonov, Phys. Rev. D 101, 094028 (2020).

[43] E. Eichten, K. Gottfried, K. Kinoshita, K. D. Lane, and T.-M. Yan, Phys. Rev. D 17, 3090 (1978); 21, 203 (1980).

[44] E. Eichten, K. Lane, and C. Quigg, Phys. Rev. D 69, 094019 (2004); Yu. S. Kalashnikova, Phys. Rev. D 72, 034010 (2005).

[45] I. V. Danilkin and Yu. A. Simonov, Phys. Rev. D 81, 074027 (2010); Phys. Rev. Lett. 105, 102002 (2010); I. V. Danilkin, V. D. Orlovsky, and Yu. A. Simonov, Phys. Rev. D 85, 034012 (2012).

[46] A. M. Badalian, L. P. Kok, M. I. Polikarpov, and Yu. A. Simonov, Phys. Rep. 82, 31 (1982).

[47] Yu. A. Simonov, Phys. Rev. D 65, 094018 (2002).

[48] Yu. A. Simonov, Phys. At. Nucl. 67, 846 (2004).

[49] Yu. A. Simonov, Phys. At. Nucl. 67, 1027 (2004).

[50] Yu. A. Simonov, Int. J. Mod. Phys. A 31, 1650104 (2016).

[51] Yu. A. Simonov, Phys. Rev. 99, 056012 (2019).

[52] M. Gell-Mann, R. L. Oakes, and B. Renner, Phys Rev. 175, 2195 (1968).

[53] A. M. Badalian, B. L. G. Bakker, and Yu. A. Simonov, Phys. Rev. 75, 116001 (2007); Yu. A. Simonov, Phys. At. Nucl. 79, 265 (2010).

[54] Yu. A. Simonov, Phys. Rev. D 99, 096025 (2019).

[55] Yu. A. Simonov, Phys. Rev. D 99, 056012 (2019); A. Di Giacomo, H. G. Dosch, V. I. Shevchenko, and Yu. A. Simonov, Phys. Rep. 372, 319 (2002); H. G. Dosh and Yu. A. Simonov, Phys. Lett. B 205, 339 (1988). 
[56] R. Garcia-Martin, R. Kaminski, J. K. Pelaez, and J. Ruiz de Elvira, Phys. Rev. Lett. 107, 072001 (2011); J. K. Pelaez, A. Rodas, and J. Ruiz De Elvira, Eur. Phys. J. C 79, 1008 (2019).
[57] T. Ledwig, J. Nieves, A. Pich, E. Ruiz Arriola, and J. Ruiz de Elvira, Phys. Rev. D 90, 114020 (2014); T. Cohen, F. J. Llanes-Estrada, J. R. Pelaez, and J. Ruiz de Elvira, Phys. Rev. D 90, 036003 (2014). 